\title{
Increasing Soybean (Glycine max L) Drought Resistance With Osmolit Sorbitol
}

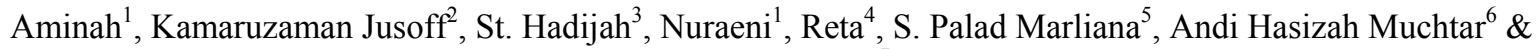 \\ Maimuna $\mathrm{Nonci}^{7}$ \\ ${ }^{1}$ Department of Agronomy, Faculty of Agriculture, Universitas Muslim Indonesia, Makassar, South Sulawesi, Indonesia \\ ${ }^{2}$ Department of Forest Production, Faculty of Forestry, Universiti Putra Malaysia, Serdang, Selangor, Malaysia \\ ${ }^{3}$ Department of Aquaculture, Faculty of Fishery and Marine Science, Universitas Muslim Indonesia, Makassar, \\ South Sulawesi, Indonesia \\ ${ }^{4}$ Politeknik Pertanian Negeri Pangkep, Pangkep, Indonesia \\ ${ }^{5}$ Department of Agricultural Technology, Faculty of Agriculture, Universitas Cokroaminoto Makassar, South \\ Sulawesi, Indonesia \\ ${ }^{6}$ Graduate Programs, Hasanuddin University, Makassar, South Sulawesi, Indonesia \\ ${ }^{7}$ Departments of Soil, Faculty of Agriculture, Universitas Hasanuddin, Makassar, South Sulawesi, Indonesia \\ Correspondence: Kamaruzaman Jusoff, Department of Forest Production, Faculty of Forestry, Universiti Putra \\ Malaysia, UPM Serdang 43400, Selangor, Malaysia. Tel: 60-3-894-67176. E-mail: kjusoff@yahoo.com
}

Received: June 5, 2013 Accepted: August 15, $2013 \quad$ Online Published: August 29, 2013

doi:10.5539/mas.v7n9p78 URL: http://dx.doi.org/10.5539/mas.v7n9p78

\begin{abstract}
Efforts to increase soybean production has been pursued for years in Indonesia through the process of intensification and extensification. Increased production through intensification of increasing grain yield per hectare, among others includes the improvement of cultivation system such as the use of cultivars that have superior resistance to drought. Increased soybean production has been through the expansion of planting areas utilizing available idle dry land. However, one of the constraints faced in dryland agriculture was the limited water supply due to low intensity of rainfall that leads to low crop production. In order to ensure that soybeans are cultivated on dry land remains capable of high production, it is necessary to physiologically engineered the soybean with an open stomata. The study was conducted in the greenhouse of Balai Penelitian Tanaman Serealia (BALITSEREAL) Maros, Sulawesi, Indonesia with a completely randomized block design $h$ factorial pattern. The first factor was the water stress stadia while the second was the amount of sorbitol osmolit concentration application. Results indicated that there was an interaction between the plant height growth and number of leaves between the water clamping time and concentration of the osmolit sorbitol. The vegetative stage especially during flowering and pod formation was inhibited when the water was clamped, but by spraying osmolit sorbitol, soybean growth interms of its height and number of leaves was enhanced. This study implies that the application of osmolit sorbitol may enhance the drought resistance of soybean growth. Future research suggested that more work should be done on the application of osmolit sorbital to other agriculture crops to increase their drought resistance in the drylands.
\end{abstract}

Keywords: drought, osmolit sorbitol, soybean, stomata, engineered physiology

\section{Introduction}

The main cause of low soybean production in Sulawesi, Indonesia is due to $65 \%$ of the soybean is grown in the dryland areas while the balance of $35 \%$ of the crop is grown in the cultivated and irrigated rice fields during the dry season. Part of the problems arises in dryland agriculture systems is the limited water supply which is very dependent on the amount and intensity of rainfall that leads to the relatively low crop production. For that reason, soybeans grown and cultivated in dry land must be able to withstand the drought and remain capable of producing high yield soybeans through the physiological engineered and regulated stomatal opening process. It has been well understood that drought stress crops may affect and lead to changes in molecular biochemistry, physiology and morphology of plants. Biochemical changes that occur may include the accumaulation of osmolit sorbitol applied to various crops and specific proteins involved in stress tolerance (Shinozaki \& Shinozaki, 2007). Soybeans 
experiencing drought will close their stomata in order to reduce the process of transpiration. However, over time the stomatal closure will result in impaired growth that leads to low crop yield production. In order to prevent the stomatal closure continues over time, it is pertinent to minimize the role of abscisic acid (ABA) in the leaves by using a local compound known as osmolit sorbitol. Plants experiencing drought will trigger the abscisic acid (ABA) biosynthesis. The process occurs due to water shortage conditions in the protoplasm where oxidation process takes place continuously (Bray, 1988). Arifin (2001) further supported that the green bean plants usually experience water shortage stress during the vegetative stage and throughout their growth cycle where abscisic acid content (ABA) increased three-folds compared to plants that were not frozen. ABA is a hormone that were synthesized in the roots and translocated to the leaves, and may also be synthesized by the guard cells themselves (Assmann \& Shimazaki, 1999). ABA contained in the leaves especially on guard cells normally control the stomatal closure (Turner, 1998). Therefore, the objective of this study was to assess the effects of applying osmolit sorbitol in increasing the soybean's resistance to drought through the stomatal physiological engineering.

\section{Methods and Materials}

Experiments were conducted in the Greenhouse Crops Research Institute for Maize and Cereals (BALITSEREAL) Maros, Sulawesi, Indonesia. The analysis was carried out in the Plant Crop Laboratory, Bogor Agricultural University (IPB), Bogor, Indonesia. The study was conducted in May to December 2012. The experiments used a designed randomized block design (RBD) in the two-factor factorial with three replicates. The time factor (Factor No. 1) was Water clamping (W), which consists of six standards namely, $\mathrm{W} 1=$ not gripped over the life of the plant, $\mathrm{W} 2=$ gripped throughout life (0-85 days), W3 = gripped from the beginning of growth (0-15 days), W4 = seized during active vegetative stadia (16-30 days), W5 = gripped the flowering-pod filling stadia (at the age 31-65 days), W6 = gripped the stadia seed maturity (age of 66-85 days). Meanwhile, the second factor (Factor No. 2) used a local compound concentration (osmolit sorbitol (S), which consists of four levels, namely S0 = No osmolit sorbitol sprayed, $\mathrm{S} 1=10 \mathrm{ml} / \mathrm{l}, \mathrm{S} 2=15 \mathrm{ml} / \mathrm{l}$, and $\mathrm{S} 3=20 \mathrm{ml} / 1$ of osmolit sorbitol sprayed. Of the two factors, a total of 24 combined treatments were obtained and each was repeated three times to obtain 72 units of the experiments. The 24 combined treatments were:

$\begin{array}{llllll}\text { W1S0 } & \text { W2S0 } & \text { W3S0 } & \text { W4S0 } & \text { W5S0 } & \text { W6S0 } \\ \text { W1S1 } & \text { W2S1 } & \text { W3S1 } & \text { W4S1 } & \text { W5S1 } & \text { W6S1 } \\ \text { W1S2 } & \text { W2S2 } & \text { W3S2 } & \text { W4S2 } & \text { W5S2 } & \text { W6S2 } \\ \text { W1S3 } & \text { W2S3 } & \text { W3S3 } & \text { W4S3 } & \text { W5S2 } & \text { W6S3 }\end{array}$

Determination of the amount of water provision (clamping) is based on $50 \%$ of the normal water demand and its distribution was based on the normal water requirements for the growth of soybeans in each period. The growth media was prepared with a mixture of soil and manure in the 4:1 ratio, which was filled in a polybag with a diameter of $20 \mathrm{~cm}$ and a height of $40 \mathrm{~cm}$ with an average weight of $8 \mathrm{~kg}$ each. Once the growth media was ready, it is filled up with water until it reached the field capacity limit. The soybean seeds were then planted as many as four points per hole. Once the soybeans reached the age of seven years old, they were then thinned untill two plants per polybag remained. With some phospohorous (P) fertilization and urea at 15 Days After Planting (DAP), the dosage of urea was given at $50 \mathrm{~kg} / \mathrm{ha}(0.66 \mathrm{~g}$ urea/polybag) and SP-36 at a dose of $90 \mathrm{~kg} / \mathrm{ha}$ (1.19 SP-36/polybag) at planting time. According to Fagi and Tangkuman (1995), the average amount of water requirement of soybean (variety Wilis) during the growing season was $325 \mathrm{~mm} / \mathrm{season}$. Using a water clamp control system, as much as $50 \%$ water was injected into the polybag to cater for the soybeans normal water requirement. The water clamping control time applied to the treatments are shown in Table 1.

Table 1. Water clamping time and osmolit sorbitol spraying of soybean

\begin{tabular}{ccccccccccccccccccc}
\hline \multirow{2}{*}{ Treatment } & \multicolumn{110}{cc}{ Implementation of water clamping time at the age (days) } \\
\cline { 2 - 7 } & 10 & 15 & 20 & 25 & 30 & 35 & 40 & 45 & 50 & 55 & 60 & 65 & 70 & 75 & 80 & 85 & Frequency \\
\hline W1 & 0 & 0 & 0 & 0 & 0 & 0 & 0 & 0 & 0 & 0 & 0 & 0 & 0 & 0 & 0 & 0 & 0 \\
W2 & $\mathrm{X}$ & $\mathrm{X}$ & $\mathrm{X}$ & $\mathrm{X}$ & $\mathrm{X}$ & $\mathrm{X}$ & $\mathrm{X}$ & $\mathrm{X}$ & $\mathrm{X}$ & $\mathrm{X}$ & $\mathrm{X}$ & $\mathrm{X}$ & $\mathrm{X}$ & $\mathrm{X}$ & $\mathrm{X}$ & $\mathrm{X}$ & 16 \\
W3 & $\mathrm{X}$ & $\mathrm{X}$ & 0 & 0 & 0 & 0 & 0 & 0 & 0 & 0 & 0 & 0 & 0 & 0 & 0 & 0 & 2 \\
W4 & 0 & 0 & $\mathrm{X}$ & $\mathrm{X}$ & $\mathrm{X}$ & 0 & 0 & 0 & 0 & 0 & 0 & 0 & 0 & 0 & 0 & 0 & 3 \\
W5 & 0 & 0 & 0 & 0 & 0 & $\mathrm{X}$ & $\mathrm{X}$ & $\mathrm{X}$ & $\mathrm{X}$ & $\mathrm{X}$ & $\mathrm{X}$ & 0 & 0 & 0 & 0 & 0 & 6 \\
W6 & 0 & 0 & 0 & 0 & 0 & 0 & 0 & 0 & 0 & 0 & 0 & $\mathrm{X}$ & $\mathrm{X}$ & $\mathrm{X}$ & $\mathrm{X}$ & $\mathrm{X}$ & 5 \\
\hline
\end{tabular}

Description: $0=$ not gripped by water; X: gripped Water

$*=$ Osmolit spraying frequency adjusted with treatment 
Osmolit sorbitol was sprayed to the 10-85 days old soybeans at the lower leaf surface once in every five days with a concentration in accordance to the frequency of spraying treatment of 2-16 times. Osmolit sorbotil was sprayed between $1000-1200 \mathrm{H}$ once in every five days, particularly at $0815-0945 \mathrm{H}, 1000-1200 \mathrm{H}$ and $1200-1400 \mathrm{H}$, despite no significant differences were observed by Ariffin (1997). The distribution and amount of water in each soybean polybags averaged at $94 \mathrm{ml}$.

\section{Results}

\subsection{Effects of Water Clamping Time and Osmolit Sorbitol on Soybean Growth Parameters}

The effects of water clamping time and osmolit sorbitol on soybean plant height, number of leaves, leave surface area, root dry weight, leaf area, dry weight of root, relative growth rate, net assimilation rate, chlorophyll content and stomatal density can be shown in Tables $2-10$, respectively.

Table 2. Effect of time, water clamping and osmolit sorbital on soybean plant height growth

\begin{tabular}{|c|c|c|c|c|c|}
\hline \multirow{2}{*}{ Water clamping time } & \multicolumn{4}{|c|}{ Osmolit sorbitol concentration ( $\mathrm{ml} / \mathrm{l}$ of water) } & \multirow{2}{*}{$\begin{array}{l}\text { HSD } \\
0.05\end{array}$} \\
\hline & $\mathrm{S}_{\mathrm{o}}(0)$ & $\mathrm{S}_{1}(10 \mathrm{ml})$ & $\mathrm{S}_{2}(15 \mathrm{ml})$ & $\mathrm{S}_{3}(20 \mathrm{ml})$ & \\
\hline (W1) without stress & 79.83 bcy & $76.00 \mathrm{cz}$ & $88.00 \mathrm{bx}$ & 99.10 ax & 10.64 \\
\hline (W2) gripped 0-85 days & $67.60 \mathrm{bx}$ & 92.85 axy & 82.83 by & $95.50 \mathrm{ax}$ & \\
\hline (W3) gripped 0-15 days & $76.67 \mathrm{cy}$ & 86.83 byz & $86.59 \mathrm{bx}$ & 103.67 ax & \\
\hline (W4) gripped $16-30$ days & 87.67 ax & 88.00 axyz & 89.65 ax & 89.67 ay & \\
\hline (W5) gripped 31-65 days & 75.83 by & $98.33 \mathrm{ax}$ & 77.83 by & 92.50 ay & \\
\hline (W6) gripped 66-85 days & $78.33 \mathrm{bx}$ & $78.67 \mathrm{bz}$ & 9500 ax & 89.83 ay & \\
\hline HSD 0.05 & 10 & & & & \\
\hline
\end{tabular}

*HSD = Honestly significant difference

Table 3. Effect of time, water clamping and osmolit sorbital on soybean number of leaves growth

\begin{tabular}{llllll}
\hline \multirow{2}{*}{ Water clamping time } & \multicolumn{4}{l}{ Osmolit Sorbitol concentration $(\mathrm{ml} / \mathrm{l}$ of water) } & HSD \\
\cline { 2 - 5 } & $\mathrm{S}_{\mathrm{o}}(0)$ & $\mathrm{S}_{1}(10 \mathrm{ml})$ & $\mathrm{S}_{2}(15 \mathrm{ml})$ & $\mathrm{S}_{3}(20 \mathrm{ml})$ & 0.05 \\
\hline (W1) without stress & $27.00 \mathrm{cy}$ & $30.50 \mathrm{bcy}$ & $36.50 \mathrm{axy}$ & $33.00 \mathrm{abz}$ & 5.75 \\
(W2) gripped 0-85 days & $28.50 \mathrm{cy}$ & $36.00 \mathrm{bx}$ & $37.50 \mathrm{abxy}$ & $42.50 \mathrm{axy}$ & \\
(W3) gripped 0-15 days & $34.50 \mathrm{bx}$ & $35.83 \mathrm{bx}$ & $32.50 \mathrm{by}$ & $43.17 \mathrm{ax}$ & \\
(W4) gripped 16-30 days & $34.50 \mathrm{ax}$ & $33.00 \mathrm{ax}$ & $35.50 \mathrm{axy}$ & $38.00 \mathrm{axyz}$ & \\
(W5) gripped 31-65 days & $28.50 \mathrm{cy}$ & $30.00 \mathrm{bcy}$ & $35.00 \mathrm{abxy}$ & $39.00 \mathrm{axy}$ & \\
(W6) gripped 66-85 days & $30.00 \mathrm{cxy}$ & $35.50 \mathrm{bcx}$ & $40.00 \mathrm{ax}$ & $37.00 \mathrm{abyz}$ & \\
\hline HSD 0.05 & 5.41 & & & & \\
\hline
\end{tabular}

*HSD $=$ Honestly significant difference

Table 4. Effect of time, water clamping and osmolit sorbital on soybean leaf area $\left(\mathrm{cm}^{2}\right)$

\begin{tabular}{llllll}
\hline \multirow{2}{*}{ Water clamping time } & \multicolumn{4}{l}{ Osmolit Sorbitol concentration $(\mathrm{ml} / \mathrm{l}$ of water $)$} & \multirow{2}{*}{ HSD 0.05 } \\
\cline { 2 - 5 } & $\mathrm{S}_{\mathrm{o}}(0)$ & $\mathrm{S}_{1}(10 \mathrm{ml})$ & $\mathrm{S}_{2}(15 \mathrm{ml})$ & $\mathrm{S}_{3}(20 \mathrm{ml})$ & \\
\hline (W1) without stress & $351.15 \mathrm{bxy}$ & $274.95 \mathrm{by}$ & $555.97 \mathrm{ax}$ & $660.45 \mathrm{axy}$ & 201.01 \\
(W2) gripped 0-85 days & $227.81 \mathrm{bx}$ & $497.45 \mathrm{ax}$ & $389.19 \mathrm{abx}$ & $392.29 \mathrm{abz}$ & \\
(W3) gripped 0-15 days & $312.56 \mathrm{ax}$ & $332.73 \mathrm{axy}$ & $492.21 \mathrm{ax}$ & $747.84 \mathrm{ax}$ & \\
(W4) gripped 16-30 days & $288.72 \mathrm{xy}$ & $284.87 \mathrm{by}$ & $286.09 \mathrm{by}$ & $557.40 \mathrm{ay}$ & \\
(W5) gripped 31-65 days & $246.69 \mathrm{bx}$ & $364.47 \mathrm{bx}$ & $508.82 \mathrm{abx}$ & $666.90 \mathrm{axy}$ & \\
(W6) gripped 66-85 days & $375.47 \mathrm{bx}$ & $438.43 \mathrm{by}$ & $785.22 \mathrm{ax}$ & $491.55 \mathrm{byz}$ & \\
\hline HSD 0.05 & 180.34 & & & & \\
\hline
\end{tabular}

*HSD $=$ Honestly significant difference 
Table 5. Effect of time, water clamping and osmolit sorbital on soybean root dry weight

\begin{tabular}{llllll}
\hline \multirow{2}{*}{ Water clamping time } & \multicolumn{3}{l}{ Sorbitol Osmolit concentration $(\mathrm{ml} / 1$ of water) } & HSD \\
\cline { 2 - 5 } & $\mathrm{S}_{\mathrm{o}}(0)$ & $\mathrm{S}_{1}(10 \mathrm{ml})$ & $\mathrm{S}_{2}(15 \mathrm{ml})$ & $\mathrm{S}_{3}(20 \mathrm{ml})$ & 0.05 \\
\hline (W1) without stress & $1103.00 \mathrm{dcz}$ & $1490.00 \mathrm{cyz}$ & $1790.00 \mathrm{by}$ & $2185.00 \mathrm{ax}$ & 239.95 \\
(W2) gripped 0-85 days & $987.00 \mathrm{bz}$ & $1070.00 \mathrm{abz}$ & $1236.00 \mathrm{az}$ & $1263.00 \mathrm{az}$ & \\
(W3) gripped 0-15 days & $1583.00 \mathrm{cy}$ & $2178.00 \mathrm{bx}$ & $2081.00 \mathrm{bx}$ & $2865.00 \mathrm{av}$ & \\
(W4) gripped 16-30 days & $1496.00 \mathrm{by}$ & $1923.00 \mathrm{ax}$ & $1821.00 \mathrm{ay}$ & $1487.00 \mathrm{by}$ & \\
(W5) gripped 31-65 days & $1725.00 \mathrm{bx}$ & $1975.00 \mathrm{ax}$ & $1185.00 \mathrm{dz}$ & $1403.00 \mathrm{cyz}$ & \\
(W6) gripped 66-85 days & $1657.00 \mathrm{cy}$ & $1876.00 \mathrm{bxy}$ & $2087.00 \mathrm{ax}$ & $2204.00 \mathrm{aw}$ & \\
\hline HSD 0.05 & 214.74 & & & & \\
\hline
\end{tabular}

*HSD $=$ Honestly significant difference

Table 6. The Effect of time, water clamping and osmolit sorbital on soybean on plant dryweight

\begin{tabular}{llllll}
\hline \multirow{2}{*}{ Water clamping time } & \multicolumn{4}{l}{ Sorbitol Osmolit concentration $(\mathrm{ml} / 1$ of water } & \multirow{2}{*}{ HSD 0.05} \\
\cline { 2 - 5 } & $\mathrm{S}_{\mathrm{o}}(0)$ & $\mathrm{S}_{1}(10 \mathrm{ml})$ & $\mathrm{S}_{2}(15 \mathrm{ml})$ & $\mathrm{S}_{3}(20 \mathrm{ml})$ & \\
\hline (W1) without stress & $15.72 \mathrm{by}$ & $16.00 \mathrm{by}$ & $19.70 \mathrm{aby}$ & $24.60 \mathrm{ax}$ & 2.95 \\
(W2) gripped 0-85 days & $12.23 \mathrm{bz}$ & $13.09 \mathrm{byz}$ & $16.12 \mathrm{ayz}$ & $16.17 \mathrm{az}$ & \\
(W3) gripped 0-15 days & $15.26 \mathrm{by}$ & $18.47 \mathrm{ax}$ & $17.01 \mathrm{by}$ & $22.12 \mathrm{axy}$ & \\
(W4) gripped 16-30 days & $19.29 \mathrm{ax}$ & $19.56 \mathrm{ax}$ & $18.56 \mathrm{ay}$ & $19.87 \mathrm{ay}$ & \\
(W5) gripped 31-65 days & $14.47 \mathrm{by}$ & $14.60 \mathrm{bz}$ & $18.47 \mathrm{ay}$ & $19.60 \mathrm{ay}$ & \\
(W6) gripped 66-85 days & $17.25 \mathrm{bx}$ & $19.30 \mathrm{bx}$ & $27.27 \mathrm{ax}$ & $24.45 \mathrm{ax}$ & \\
\hline HSD 0.05 & 2.64 & & & &
\end{tabular}

$* \mathrm{HSD}=$ Honestly significant difference

Table 7. Effect of time, water clamping and osmolit sorbital on soybean relative growth rate ( $\mathrm{mg} \mathrm{mg}^{-1}$.days)

\begin{tabular}{llllll}
\hline \multirow{2}{*}{ Water clamping time } & \multicolumn{4}{c}{ Osmolit Sorbitol concentration (ml/1 of water) } & \multirow{2}{*}{ HSD 0.05 } \\
\cline { 2 - 5 } & $\mathrm{S}_{\mathrm{o}}(0)$ & $\mathrm{S}_{1}(10 \mathrm{ml})$ & $\mathrm{S}_{2}(15 \mathrm{ml})$ & $\mathrm{S}_{3}(20 \mathrm{ml})$ & \\
\hline (W1) without stress & $26.55 \mathrm{bx}$ & $25.68 \mathrm{bxy}$ & $31.33 \mathrm{bx}$ & $48.11 \mathrm{ax}$ & 15.31 \\
(W2) gripped 0-85 days & 14.29 ay & 14.93 ay & 20.57 ay & 22.52 ay & \\
(W3) gripped 0-15 days & 22.18 axy & 22.62 axy & 23.76 ay & 30.93 ay & \\
(W4) gripped 16-30 days & 12.11 ay & 19.04 ay & 12.96 ayz & $26.55 \mathrm{ay}$ & \\
(W5) gripped 31-65 days & 13.45 by & 14.78 by & 28.36 aby & $37.88 \mathrm{ax}$ & \\
(W6) gripped 66-85 days & 29.40 bxy & $30.58 \mathrm{bx}$ & $40.18 \mathrm{abx}$ & $47.82 \mathrm{ax}$ & \\
\hline HSD 0.05 & 13.73 & & & & \\
\hline
\end{tabular}

*HSD = Honestly significant difference 
Table 8. Effect of time, water clamping and osmolit sorbital on soybean net assimilation rate $\left(\mathrm{mg} \cdot \mathrm{cm}^{-2} \mathrm{days} \mathrm{s}^{-1}\right)$

\begin{tabular}{llllll}
\hline \multirow{2}{*}{ Water clamping time } & \multicolumn{3}{c}{ Osmolit Sorbitol concentration $(\mathrm{ml} / 1$ of water $)$} & \multirow{2}{*}{ HSD 0.05} \\
\cline { 2 - 4 } & $\mathrm{S}_{\mathrm{o}}(0)$ & $\mathrm{S}_{1}(10 \mathrm{ml})$ & $\mathrm{S}_{2}(15 \mathrm{ml})$ & $\mathrm{S}_{3}(20 \mathrm{ml})$ & \\
\hline (W1) without stress & $0.64 \mathrm{ax}$ & $1.35 \mathrm{ax}$ & $0.89 \mathrm{az}$ & $1.31 \mathrm{ay}$ & 0.77 \\
(W2) gripped 0-85 days & $0.04 \mathrm{by}$ & $0.39 \mathrm{ay}$ & $0.39 \mathrm{ax}$ & $0.44 \mathrm{ayz}$ & \\
(W3) gripped 0-15 days & $0.70 \mathrm{ax}$ & $0.84 \mathrm{ax}$ & $0.93 \mathrm{ax}$ & $1.14 \mathrm{ayz}$ & \\
(W4) gripped 16-30 days & $0.39 \mathrm{bcx}$ & $0.14 \mathrm{cy}$ & $0.98 \mathrm{abx}$ & $1.20 \mathrm{ay}$ & \\
(W5) gripped 31-65 days & $0.42 \mathrm{bx}$ & $0.46 \mathrm{by}$ & $0.45 \mathrm{bx}$ & $1.88 \mathrm{axy}$ & \\
(W6) gripped 66-85 days & $0.56 \mathrm{bx}$ & $0.87 \mathrm{bxy}$ & $1.10 \mathrm{bx}$ & $2.17 \mathrm{ax}$ & \\
\hline HSD 0.05 & 0.69 & & & \\
\hline
\end{tabular}

$* \mathrm{HSD}=$ Honestly significant difference

Table 9. Effect of time, water clamping and osmolit sorbital on soybean chlorophyll content (g/g)

\begin{tabular}{llcccc}
\hline & \multicolumn{5}{c}{ Osmolit Sorbitol concentration (ml/1 of water) } \\
\cline { 2 - 5 } Water clamping time & 0 & 10 & 15 & 20 & HSD 0.05 \\
& \multicolumn{1}{c}{$(\mathrm{S} 0)$} & $(\mathrm{S} 1)$ & $(\mathrm{S} 2)$ & $(\mathrm{S} 3)$ & \\
\hline W1 without stress & $3.00 \mathrm{cxy}$ & $3.54 \mathrm{bx}$ & $3.66 \mathrm{bx}$ & $4.83 \mathrm{ax}$ & 0.43 \\
W2 gripped 0-85 days & $2.33 \mathrm{cz}$ & $4.36 \mathrm{bw}$ & $3.68 \mathrm{cx}$ & $3.21 \mathrm{az}$ & \\
W3 gripped 0-15 days & $3.35 \mathrm{awx}$ & $3.40 \mathrm{axy}$ & $3.67 \mathrm{ax}$ & $3.47 \mathrm{ayz}$ & \\
W4 gripped 16-30 days & $2.72 \mathrm{cy}$ & $2.94 \mathrm{cyz}$ & $3.59 \mathrm{bxy}$ & $4.67 \mathrm{ax}$ & \\
W5 gripped 31-65 days & $2.82 \mathrm{ay}$ & $3.05 \mathrm{ay}$ & $3.06 \mathrm{ay}$ & $3.81 \mathrm{ay}$ & \\
W6 gripped 66-85 days & $3.68 \mathrm{cw}$ & $2.60 \mathrm{cz}$ & $3.19 \mathrm{by}$ & $3.60 \mathrm{ayz}$ & \\
\hline HSD 0.05 & 0.39 & & & \\
\hline
\end{tabular}

$*$ HSD $=$ Honestly significant difference

Table 10. Effect of time, water clamping and osmolit sorbital on soybean stomatal density

\begin{tabular}{|c|c|c|c|c|c|}
\hline \multirow{3}{*}{ Water clamping time } & \multicolumn{4}{|c|}{ Osmolit Sorbitol concentration (ml/l of water) } & \multirow{3}{*}{ HSD 0.05} \\
\hline & 0 & 10 & 15 & 20 & \\
\hline & $(\mathrm{S} 0)$ & $(\mathrm{S} 1)$ & $(\mathrm{S} 2)$ & (S3) & \\
\hline W1 without stress & $42.12 \mathrm{ax}$ & 44.15 axy & $48.10 \mathrm{ax}$ & 52.75 ax & 8.06 \\
\hline W2 gripped 0-85 days & $22.17 \mathrm{bz}$ & 27.96 aby & $29.87 \mathrm{abz}$ & 32.75 ay & \\
\hline W3 gripped 0-15 days & 27.25 byz & $24.15 \mathrm{bz}$ & $25.00 \mathrm{bz}$ & 38.92 ay & \\
\hline W4 gripped16-30 days & $26.75 \mathrm{az}$ & $21.83 \mathrm{az}$ & $22.09 \mathrm{az}$ & 34.69 ay & \\
\hline W5 gripped31-65 days & 31.17 bxy & 33.78 aby & 35.78 aby & 40.42 ay & \\
\hline W6gripped 66-85 days & 33.85 bxy & 48.19 ax & $51.00 \mathrm{ax}$ & $49.50 \mathrm{ax}$ & \\
\hline HSD 0.05 & 7.58 & & & & \\
\hline
\end{tabular}

Description: The average value followed by the letters $a, b, c$ significantly different in each row and $x, y, z$ in each column

3.2 Effects of Water Clamping Time and Osmolit Sorbitol on the Content of Abscisic Acid (ABA) and Number of Pods, Pod Weight and Seed Weight per Plant and Weight of 100 Seeds

ABA is a plant hormone that acts as a natural barrier formed by itself due to the lack of water and environmental stress. Based on the analysis of clamping turning times, there was an effect of water on the ABA content in the 
leaves of green soybean plants, whereas spraying osmolit sorbitol provided no significant interactions as demonstrated in Tables 11-13. Results indicated that the time of water clamping had very significant effects on the weight of 100 seeds while spraying osmolit sorbitol had a no significant interaction with water clamping time as shown in Table 13.

Table 11. Effect of water clamping time on ABA content (ug/g)

\begin{tabular}{lllll}
\hline \multirow{2}{*}{ Water clamping time } & \multicolumn{4}{c}{ Average content of ABA (ug/g) } \\
\cline { 2 - 5 } & 35 DAP & 50 DAP & 65 DAP & 80 DAP \\
\hline W1 without stress & $0.09 \mathrm{~b}$ & $0.11 \mathrm{~b}$ & $0.12 \mathrm{bc}$ & $0.13 \mathrm{c}$ \\
W2 gripped 0-85 days & $0.25 \mathrm{a}$ & $0.36 \mathrm{a}$ & $0.50 \mathrm{a}$ & $0.62 \mathrm{a}$ \\
W3 gripped 0-15 days & $0.09 \mathrm{~b}$ & $0.10 \mathrm{~b}$ & $0.10 \mathrm{c}$ & $0.12 \mathrm{c}$ \\
W4 gripped 16-30 days & $0.08 \mathrm{~b}$ & $0.08 \mathrm{~b}$ & $0.10 \mathrm{c}$ & $0.12 \mathrm{c}$ \\
W5 gripped 31-65 days & $0.21 \mathrm{a}$ & $0.34 \mathrm{a}$ & $0.48 \mathrm{a}$ & $0.10 \mathrm{c}$ \\
W6 gripped 66-85 days & $0.09 \mathrm{~b}$ & $0.10 \mathrm{~b}$ & $0.25 \mathrm{~b}$ & $0.32 \mathrm{~b}$ \\
\hline HSD 0.05 & 0.05 & 0.06 & 0.14 & 0.12 \\
\hline
\end{tabular}

$*(\mathrm{DAP})=$ Days After Planting, ${ }^{*} \mathrm{HSD}=$ Honestly significant difference

Table 12. Effect of water clamping time and osmolit sorbitol on soybean total pods, pod weight and weight seeds

\begin{tabular}{|c|c|c|c|c|c|}
\hline \multicolumn{6}{|c|}{ Number of soybean pods } \\
\hline Water clamping time & \multicolumn{4}{|c|}{ Osmolit sorbitol concentration ( $\mathrm{ml} / \mathrm{l}$ of water) } & \multirow{2}{*}{ HSD 0.05} \\
\hline & $\mathrm{S}_{\mathrm{o}}(0)$ & $\mathrm{S}_{1}(10 \mathrm{ml})$ & $\mathrm{S}_{2}(15 \mathrm{ml})$ & $\mathrm{S}_{3}(20 \mathrm{ml})$ & \\
\hline (W1) without stress & 21.17 bxy & 23.32 bwx & 24.50 by & $37.50 \mathrm{ax}$ & 3.97 \\
\hline (W2) gripped $0-85$ days & $15.50 \mathrm{bz}$ & $17.17 \mathrm{abz}$ & 20.84 ay & $21.83 \mathrm{az}$ & \\
\hline (W3) gripped $0-15$ days & 22.17 bxy & $23.49 \mathrm{abx}$ & 22.50 by & 26.50 ay & \\
\hline (W4) gripped 16-30 days & 19.34 ayz & 21.16 axy & 21.67 ay & 26.52 ay & \\
\hline (W5) gripped 31-65 days & 19.00 ayz & 18.51 abyz & $14.83 \mathrm{bz}$ & $18.00 \mathrm{abz}$ & \\
\hline (W6) gripped 66-85 days & $24.33 \mathrm{cx}$ & $26.17 \mathrm{cw}$ & $31.50 \mathrm{ax}$ & 28.82 ay & \\
\hline HSD 0,05 & 3.74 & & & & \\
\hline \multicolumn{6}{|c|}{ Observation Pods Weight (g) Soybean Plants } \\
\hline (W1) without stress & 5.64 bxy & $7.82 \mathrm{ax}$ & $7.78 \mathrm{ax}$ & $11.28 \mathrm{ax}$ & 2.25 \\
\hline (W2) gripped 0-85 days & 3.95 by & $4.15 \mathrm{bz}$ & 4.85 abyz & $5.21 \mathrm{az}$ & \\
\hline (W3) gripped 0-15 days & $6.70 \mathrm{ax}$ & $7.05 \mathrm{ax}$ & 7.00 axy & 6.98 ayz & \\
\hline (W4) gripped 16-30 days & 5.05 axy & 6.68 axy & 5.74 axyz & 6.58 ayz & \\
\hline (W5) gripped 31-65 days & 4.92 axy & 4.60 ayz & $4.46 \mathrm{az}$ & 6.66 ay & \\
\hline (W6) gripped 66-85 days & $6.79 \mathrm{bx}$ & $7.20 \mathrm{bx}$ & $7.55 \mathrm{bx}$ & 8.26 ay & \\
\hline HSD 0,05 & 2.12 & & & & \\
\hline \multicolumn{6}{|c|}{ Observations Weight Seeds / Crops (g) Soybean } \\
\hline (W1) without stress & 3.84 bxy & $5.15 \mathrm{bx}$ & 5.45 bxy & 8.19 ax & 1.99 \\
\hline (W2) gripped 0-85 days & 2.28 ay & 2.70 ay & $2.85 \mathrm{az}$ & 3.87 ayz & \\
\hline (W3) gripped 0-15 days & $4.77 \mathrm{ax}$ & 5.11 ax & 5.00 axy & $6.65 \mathrm{ax}$ & \\
\hline (W4) gripped 16-30 days & 3.30 axy & 4.60 axy & 3.72 ay & 4.53 ay & \\
\hline (W5) gripped 31-65 days & 3.45 axy & 3.16 axy & 3.22 ay & 4.23 ay & \\
\hline (W6) gripped 66-85 days & 4.74 ax & $4.82 \mathrm{ax}$ & 5.21 axy & 5.80 ay & \\
\hline HSD 0.05 & 1.87 & & & & \\
\hline
\end{tabular}

*HSD $=$ Honestly significant difference 
Table 13. The effects of clamping time and osmolit sorbitol on the weight of 100 seeds

\begin{tabular}{lllllll}
\hline & \multicolumn{7}{l}{ Concentration of osmolit sorbitol (ml/l of water) } & & HSD \\
\cline { 2 - 5 } Water Clamping Time & 0 & 10 & 15 & 20 & Average & 0.05 \\
& $(\mathrm{~S} 0)$ & $(\mathrm{S} 1)$ & $(\mathrm{S} 2)$ & $(\mathrm{S} 3)$ & & $10.31 \mathrm{a}$ \\
\hline (W1) without stress & 10.35 & 10.61 & 10.45 & 9.81 & 1.37 \\
(W2) gripped 0-85 days & 8.83 & 9.14 & 8.93 & 9.3 & $9.05 \mathrm{~b}$ & \\
(W3) gripped 0-15 days & 9.28 & 11.26 & 11.98 & 11.78 & $11.07 \mathrm{a}$ & \\
(W4) gripped 16-30 days & 10.66 & 10.6 & 9.94 & 9.21 & $10.10 \mathrm{a}$ & \\
(W5) gripped 31-65 days & 10.26 & 9.49 & 9.72 & 9.19 & $9.67 \mathrm{~b}$ & \\
(W6) gripped 66-85 days & 10.63 & 9.93 & 8.65 & 10.8 & $10.00 \mathrm{a}$ & \\
\hline
\end{tabular}

Description: Average value, followed by the letters a, b, c significantly different by HSD test at level $\alpha=0.05$

\section{Discussion}

The results of the study clearly showed that the growth of soybean was significantly affected by the availability of water. This is because water is deemed necessary for the metabolic activities of the plant for a paired cell growth and development. Lack of water during the vegetative stadia and flowering until pod formation showed the lowest period of growth. When compared with normal plants that obtain sufficient water, lack of water at each stadia results in a decrease of crop growth. Aminah (2007) found that soybean with a water stress at 150 $\mathrm{mm} /$ season (below the normal requirement) showed highly significant growth differences with soybean getting water at $300 \mathrm{~mm} / \mathrm{season}$. The most sensitive and critical growth phase to drought is during the vegetative stadia, flowering and pod formation, as evidenced by the low growth measurements of their vegetative organs. Simillar results were obtained by Boyer (1996) where he observed that the growth of soybean pod formation wash and water shortages could affect the later low seed yield obtained. Aminah (2011) revealed that a strong osmotic regulation can improve the adaptability of plants during drought where the water supply is limited. Apparently, spraying osmolit sorbitol in soybeans when experiencing water shortages can increase its growth resistance to drought. This has been proven with water clamping time and spraying with osmolit sorbitol effects both in terms of plant height (for all observation time) and number of leaves (50 observations and 65 DAP).

In the process of photosynthesis, stomatal opening is an important part of the plant where the process of gas or water vapor diffusion into and out of plant tissue occurs. The process of opening and closing of stomata is one of the obstacles that occur in the diffusion process. The process of opening and closing of stomata is usually affected by stomatal guard cell turgor where it closes under the conditions of water shortage (Turner, 1998).

The results of the analysis shows that spraying osmolit sorbitol to soybeans under stress experiencing water shortages can increase its resistance to water shortages in terms of plant height and total number of leaves. Osmolit sorbitol can also control the cell's humidity where the cell turgor can be maintained with sufficient water for higher yields.

\section{Conclusion}

Soybean growth (W4) especially its plant height, leaf number, leaf area, root dry weight, content chlorophyll, and variable production is heavily reliable and sensitive to the availability of water. An osmolit sorbitol concentration of $20 \mathrm{ml} / 1$ sprayed on the soybeans increased its water drought resistance by controlling its physiological stomatal activity at various stadia growth and soybean yield. This study implies that the local compound osmolit sorbitol can enhance the soybean growth in drought resistance dryland areas.

\section{References}

Aminah, M. (2007). Respons beberapa varietas tanaman kedelai (Glycine max L) pada kondisi cekaman air (Penelitian mandiri 2007), hal 24-32 (In Indonesian).

Aminah, M. (2011). Respon pertumbuhan dan hasil tanaman kedelai (Glycine max L) pada cekaman kekeringan dengan menggunakan pupuk organic. Jurnal Ilmiah AKTUALITA. Kopertis Wilayah IX. Vol.III. Edisi ke-6 Des 2011 (In Indonesian).

Ariffin. (1997). Pengaruh sorbitol terhadap pertumbuhan dan hasil tanaman kacang hijau. Fakultas Pertanian Universita Brawijaya Malang, hal 30-48 (In Indonesia). 
Ariffin. (2001). Respons tanaman kacang hijau (Vigna radiata L) terhadap kekurangan air dan upaya meningkatkan ketahanan tanaman pada kondisi kekeringan. Program PascaSarjana Universitas Airlangga Surabaya, hal 10-32 (In Indonesian).

Assmann, S. M., \& Shimazaki, K. (1999). The multisensory guard cell. stomatal responses to blue light and abscisid acid (ABA). Plant Physiol, 119, 809-816. http://dx.doi.org/10.1104/pp.119.3.809

Boyer, S. J. (1996). Advances in drought tolerance in plants. Adv. in Agron, 56, $187-213$. http://dx.doi.org/10.1016/S0065-2113(08)60182-0

Bray, E. (1988). Drought and ABA-Induced changes in polypeptide and mRNA accumulation in tomato leaves. Plant Physiol, 88, 1210-1214. http://dx.doi.org/10.1104/pp.88.4.1210

Fagi, A. M., \& Tangkuman, F. (1995). Pengelolaan air untuk pertanaman kedelai pusat penelitian dan pengembangan tanaman pangan. Bogor, 135-151 (In Indonesian).

Shinozaki, K., \& Shinozaki, K. Y. (2007). Gene networks involved in drought stress response and tolerance. J. Exp Bot, 58(2), 221-227. http://dx.doi.org/10.1093/jxb/erl164

Turner, C. N. (1998). Crop Water Deficit. A decade of Progress. Adv. in Agron, 39, 1-39. http://dx.doi.org/10.1016/S0065-2113(08)60464-2

\section{Copyrights}

Copyright for this article is retained by the author(s), with first publication rights granted to the journal.

This is an open-access article distributed under the terms and conditions of the Creative Commons Attribution license (http://creativecommons.org/licenses/by/3.0/). 